\title{
An Adaptive Genetic Algorithm for Digital Facility Layout Optimization Under the Multi-Species and Variable-Batch Production Mode
}

\author{
Daoguo Li, Zhaoxia Chen and Rong Zhao*
}

Management school, Hangzhou Dianzi University, Hangzhou, 310018, China

\begin{abstract}
For the NP-hard characteristic of facility layout problem (FLP) and its importance to industry, many optimal and heuristic algorithms have been designed to solve the problem. But when optimizing the production, with fixed probabilities, traditional GA has its flaws with slow convergence speed and the less-than ideal accuracy of the optimal solution. According to the characteristics of multi-species and variable-batch production mode, this paper analyzed those weak points and proposed an improved adaptive genetic algorithm with the objective of minimizing the material handling cost. This genetic algorithm has adaptive probabilities based on the fitness. It can keep the diversity and excellence of the genes. The proposed model of GA also has been tested and verified by simulation with MTALAB. According to the results, it shows that the proposed approach improved the convergence speed and the accuracy of the optimal solution and can help the factory make right decision.
\end{abstract}

Keywords: Facility layout, Genetic Algorithms, Material Handling Cost, Multi-Species and Variable-Batch.

\section{INTRODUCTION}

With the development of economic globalization, production mode changes from the state of mass production with single variety to multi-species and variable-batch products, which put forward higher demand to the enterprises. The importance of good facility planning can be shown from that $20 \%-50 \%$ of the total operating expense during manufacturing can be attributed to material handling cost, and $10 \%-30 \%$ of the material handling cost can be saved by good facility planning. Therefore, it is great realistic significant for the search of the design of facility planning [1].

Zhou Er-min and Chen Ke-lou [2] used systematic layout planning (SLP) to design the layout of an iron and steel plants which is based on the data of the workshops, auxiliary plants and power plants and so on. SLP was proposed by Richard Muthur in 1973 [3]. To some extent, SLP can solve the facility layout problem, but it is subjective when deciding the relationship strength between the workshops and when the size of the problem expands, it is not effective enough. In 1976, Sabni and Gonzalc [4] proved that facility layout problem is a typical NP-hard problem. Then heuristic algorithms have been applied in this field, such as genetic algorithm, simulated annealing algorithms and tabu search. Long Qiao [5] proposed a genetic algorithm for the optimization model with the objectives of minimizing material handling cost and space cost. Ramazan Sahin [6] developed a simulated annealing algorithm to solve a bi-objective facility layout problem. Kuan Yew Wong and Phen Chiak See [7] proposed a hybrid ant colony optimization algorithm solving QAP. Ge

*Address correspondence to this author at the Management school, Hangzhou Dianzi University, Hangzhou, 310018, China; Tel: 13065735516, 15869026320; E-mal: czxfighting@163.com netic algorithm is random and global searching optimizing algorithm based on based on the mechanism of "survival of the fittest" of biological evolutionary theory and the population genetics principle, which is also highly parallel and robust [8]. But the convergence speed of traditional GA is slow and that the accuracy of the optimal solution is not ideal. To overcome these shortcomings, this article presents a solution for layout problem with the object of minimizing material handling cost according to the physical dimension of the facilities and their logistical relationships by analyzing the characteristics of multi-species and variable-batch production mode that can be defined as follows: diversification of manufacturing objects, the diversification of machines, rapid changes of the market. etc.

\section{PROBLEM DESCRIPTION AND MODEL BUILD- ING PROBLEM DESCRIPTION}

The entire facility layout problem can be defined as the reasonable organization of facilities and resources within the physics space and subject to certain constraints in order to optimize the production progress and reduce manufacture cost. It can be described by Eq. (1) as below.

Layout $=\{F, S, C, O\}$

F: facilities to be planned; S: Space of the workshop; C: certain constraints; O: Layout object

In this paper there are some hypotheses as follows for the solution of such a problem:

H1: All the facilities and workshops are in rectangle and ignore their details;

$\mathrm{H} 2$ : Only one product can be produced on one machine at the same time; 


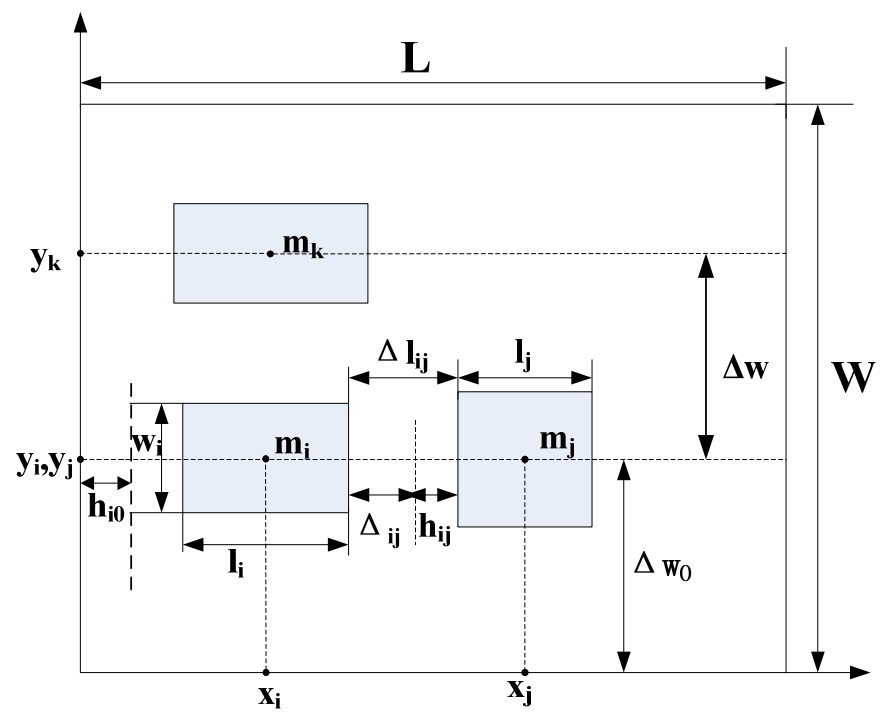

Fig. (1). The parameters and reference line of mathematical model.

H3: The ordinate values of the centre for the machines in the same row must be the same;

H4: The height of the facilities shorter than the factory's.

\section{MATHEMATICAL MODEL}

The objective of this facility layout problem is to minimize the material handling cost between the facilities in the workshop which is determined by the production volumes, distance and the material handling frequency between facilities. To express the mathematical model clearly, the parameters and reference line of mathematical model is shown in Fig. (1) and the following notations will be used in the model.

Indices:

Parameters:

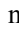

$m_{i}$

$\left(x_{1}, y_{l}\right)$

$\mathrm{R}$

$l_{i}$

$w_{i}$

$\Delta w_{0}$

$\Delta l_{i j} \quad$ distance between facility $\mathrm{i}$ and $\mathrm{j}$ in the horizontal direction;

$\Delta w$

$h_{i j}$

$\Delta_{i j}$ machine indices; $i=1,2, \ldots \ldots, n$

line indices; $r=1,2, \ldots \ldots, \mathrm{R}$

total number of the facilities;

sign of facility $\mathrm{i}$;

coordinate of geometric center of facility $\mathrm{i}$;

total number of the lines;

length of facility $\mathrm{j}$;

width of facility i;

constant distance between the first line and the workshop wall( $\mathrm{X}$ axis);

constant line space in vertically;

minimum spacing that facilities $\mathrm{i}$ and $\mathrm{j}$ must keep in the

horizontal direction; direction;

$d_{i j} \quad$ rectilinear distance between facilities $\mathrm{i}$ and $\mathrm{j}$;

$C_{i j} \quad$ unit material handling cost between facilities $\mathrm{i}$ and $\mathrm{j}$;

$f_{i j} \quad$ number of loads required between facilities $\mathrm{i}$ and $\mathrm{j}$.

Decision variables:

$v_{i r} \quad v_{i r}=1$ when facility i located in the line of number $\mathrm{r}$, otherwise $v_{i r} \mathrm{~V}_{\mathrm{ir}=0}$

According to the definition of the parameters above, the total cost function is defined as Fig. (2):

Target function:

$\min L C=\sum_{i=1}^{n-1} \sum_{j=i+1}^{n} c_{i j} \times d_{i j} \times f_{i j}$

Subject to:

$\sum_{\mathrm{r}=1}^{\mathrm{R}} \mathrm{v}_{\mathrm{ir}}=1$

$\Delta l_{i j}=h_{i j}+\Delta_{i j}$

$x_{i} \geq 0, y_{i} \geq 0, \Delta_{i j} \geq 0, \Delta l_{i j} \geq 0$

$\left|x_{j}-x_{i}\right| \geq \Delta l_{i j}+\frac{1}{2}\left(l_{i}+l_{j}\right)$

$\left|y_{j}-y_{i}\right| \geq \Delta w_{i j}+\frac{1}{2}\left(w_{i}+w_{j}\right)$

$\max \left\{x_{i}\right\}-\min \left\{x_{i}\right\}+\frac{1}{2}\left(l_{i}+l_{j}\right) \leq L$ 


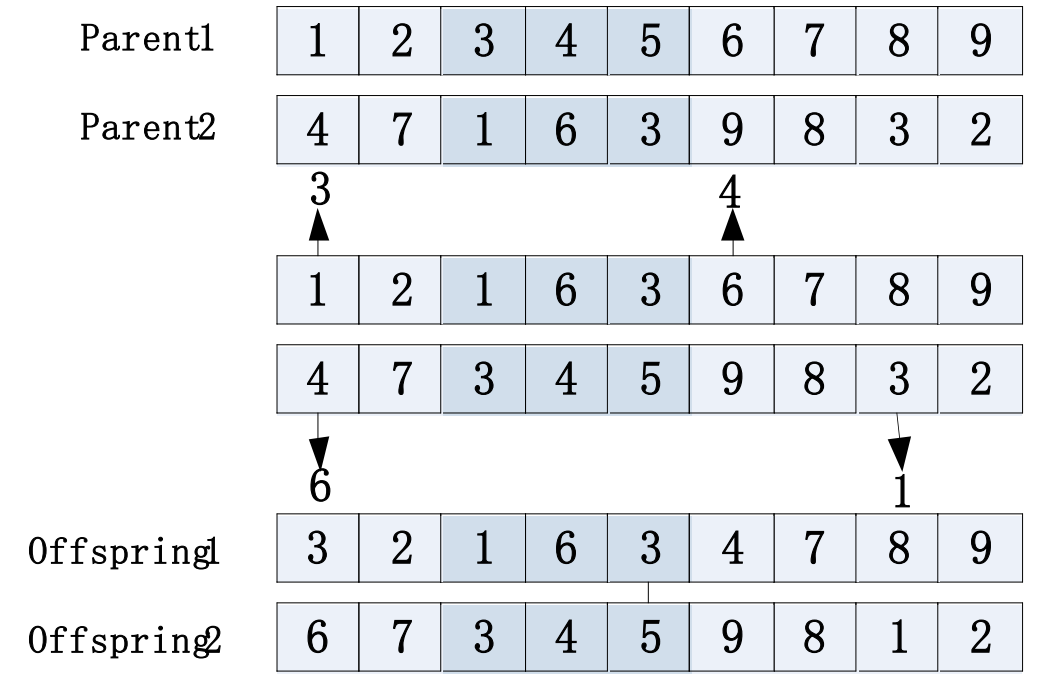

Fig. (2). Crossover operator.

$$
\mathrm{y} \_\max =\max \left\{y_{i}+\frac{1}{2} w_{i}\right\}
$$

\section{GENETIC ALGORITHMS}

\subsection{Encoding of Chromosome}

Encoding of the chromosome is vital for the GA operators and final result. In this case, the chromosome includes two parts which are the facility numbers $m_{i}$ and the clear spacing QWE $\Delta_{i}$. The chromosome is expressed in Eq. (10).

$$
\begin{array}{r}
\text { Chromosome }_{k}=\left\{\left[m_{1}, m_{2}, \cdots, m_{i}, \cdots, m_{n}\right],\right. \\
\left.\left[\Delta_{1}, \Delta_{2}, \cdots, \Delta_{i}, \cdots, \Delta_{n}\right]\right\}
\end{array}
$$

\subsection{Fitness Function}

According to the rule that "the smaller cost, the more profits", in this case, the proposed fitness function is the reciprocal of LC shown in Eq. (2). Meanwhile, this paper adopts a line wrap strategy, so it is only possible for the facility to cross the boundary in the direction of $\mathrm{Y}$-axis. Then the punishment functions with $P_{k}$ shows as below:

$$
P_{k}= \begin{cases}0, & y_{-} \max \leq W \\ \left(y_{-} \max -W\right), & y_{-} \max \geq W\end{cases}
$$

The fitness function is defined as:

$$
F\left(\text { Chromosome }_{k}\right)=P_{k} \times \frac{1}{L C(k)}
$$

\subsection{Selection Operator}

This research put forward a selecting operator combined with optimal conservation method and greedy strategy. Firstly find out the best one in the population and keep it into the next generation. Secondly design the greedy strategy based on the probability, which is decided by the fitness of the chromosome, and then those reminding individuals enter into the next generation according to $P_{s}$ as below:

$$
P_{s}=\frac{\operatorname{eval}_{\left(\text {Chromosome }_{k}\right)-\min (F)}}{\max (F)-\min (F)}
$$

\subsection{Crossover Operator}

In the crossover operator, crossover probability here decided by Eq. (14) is adaptive. $P_{c 1}$ and $P_{c 2}$ are the minimum and maximum crossover probability . The proposed crossover operator is different for the two parts of the chromosome. This paper use Goldberg's Partially Mapped Crossover shown in Fig. (2) for the first part (facility code) and the arithmetic crossover has shown in Eq. (15) and Eq. (16)for the other part (clear spacing).

$$
\begin{aligned}
& P_{c}=P_{c 1}+\frac{\left(P_{c 2}-P_{c 1}\right) \times\{F-\min (F)\}}{\max (F)-\min (F)} \\
& A^{t+1}=\beta \times A^{t}+(1-\beta) \times B^{t} \\
& B^{t+1}=\beta \times B^{t}+(1-\beta) \times A^{t}
\end{aligned}
$$

\subsection{Mutation Operator}

This paper adopts the adaptive mutation probability decided by Eq. (17). $P_{m 1}$ and $P_{m 1}$ are the minimum and maximum crossover probability. For the first part of the chromosome, choose two genes randomly and exchange them. For the second part, choose a non-zero gene e.g. $\Delta_{i}$. With a given integer ' $r$ ', it can create $r$ new chromosomes according to Eq. (18) and Eq. (19). Then calculate their fitness and pick the best one to replace $\Delta_{i}$.

$$
\begin{aligned}
& P_{m}=P_{m 1}+\frac{\left(P_{m 2}-P_{m 1}\right) \times\{F-\min (F)\}}{\max (F)-\min (F)} \\
& \Delta_{i}^{1}=\frac{\Delta_{i}}{r}, \lambda=2,3, \cdots, 2 r \\
& \Delta_{i}^{\lambda}=\Delta_{i}^{\lambda-1}+\frac{\Delta_{i}}{r}
\end{aligned}
$$




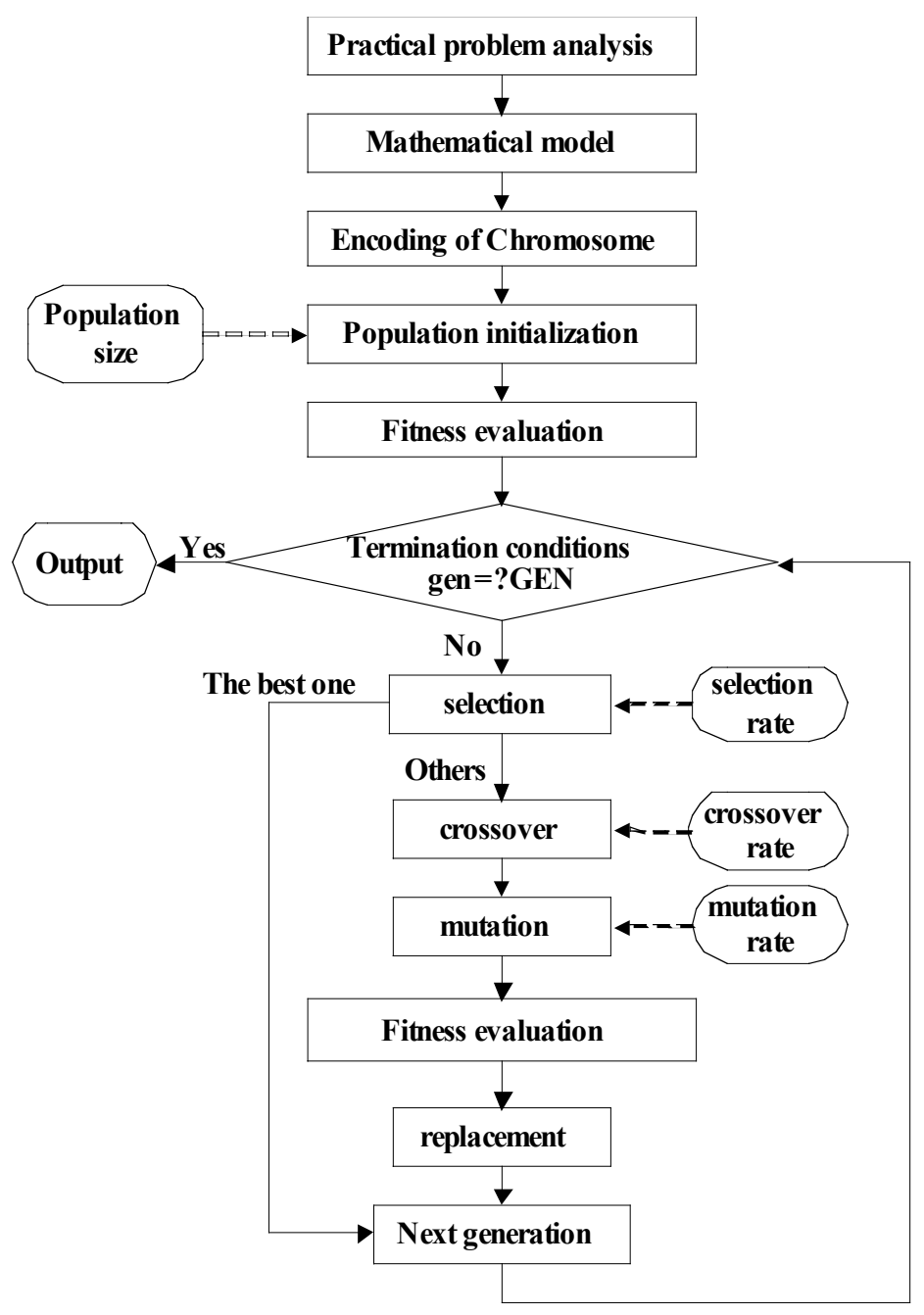

Fig. (3). Framework of Gas.

Table 1. Parameters of facilities

\begin{tabular}{|c|c|c|c|c|c|c|c|c|c|c|}
\hline Machine & $\mathbf{1}$ & $\mathbf{2}$ & $\mathbf{3}$ & $\mathbf{4}$ & $\mathbf{5}$ & $\mathbf{6}$ & $\mathbf{7}$ & $\mathbf{8}$ & $\mathbf{9}$ & $\mathbf{1 0}$ \\
\hline \hline 1 & 1 & 1.2 & 1.5 & 0.5 & 0.8 & 1 & 0.9 & 1 & 0.8 & 2 \\
\hline $\mathrm{w}$ & 1.2 & 2 & 1 & 0.5 & 1 & 1.2 & 1.1 & 1.3 & 1.2 & 1.5 \\
\hline
\end{tabular}

\subsection{Framework of GA}

As we have discussed above, the genetic algorithm has steps such as encoding, selection, and crossover and so on. In this paper, we have the steps below:

(1) After analyzing the practical problem and building the math model, encoding the chromosomes with the facility number and the clear spacing;

(2) Initialize the population randomly with the population size decided before;

(3) Calculate the fitness of the generation;

(4) Decide whether the generation meet the limitation of the termination condition. If yes, to step 10, else to step (5);

(5) Calculate the fitness of the generation and pick the best one into next generation directly, then calculate the $P_{s}$ of others and select them according to their individual probability;

(6) Crossover and mutate the individuals based on their crossover probability and mutation probability.

(7) Calculate the fitness of the generation, if it is better than their parents, and then replace them to the next generation. Then to step (4);

(8) Output the result.

The process can be described as Fig. (3).

\section{NUMERICAL EXAMPLE}

This example includes ten facilities and a workshop $10 \mathrm{~m}$ long and $8 \mathrm{~m}$ wide. The parameters of those facilities are shown in Table 1 . The matrix of $f_{i j}, c_{i j}$ and $h_{i j}$ are shown as follows: 
Table 2. Comparison of the output with POPSIZE $=\mathbf{5 0}$

\begin{tabular}{|c|c|c|c|}
\hline ALGORITHM APPLIED & Elapsed Time (Seconds) & Convergence Generation & Material Handling Cost \\
\hline \hline traditional GA & 10.265621 & 190 & 2130 \\
\hline proposed GA & 12.615298 & 170 & 2050 \\
\hline
\end{tabular}

Table 3. Comparison of the output with POPSIZE $=100$

\begin{tabular}{|c|c|c|c|}
\hline Algorithm Applied & Elapsed Time (Seconds) & Convergence Generation & Material Handling Cost \\
\hline \hline traditional GA & 18.692157 & 190 & 2090 \\
\hline proposed GA & 24.300940 & 180 & 2030 \\
\hline
\end{tabular}

Table 4. Comparison of the output with POPSIZE $=200$

\begin{tabular}{|c|c|c|c|}
\hline Algorithm Applied & Elapsed Time (Seconds) & Convergence Generation & Material Handling Cost \\
\hline \hline traditional GA & 37.877463 & 130 & 2050 \\
\hline proposed GA & 46.629072 & 100 & 1970 \\
\hline
\end{tabular}

Table 5. Comparison of the Output with POPSIZE $=300$

\begin{tabular}{|c|c|c|c|}
\hline Algorithm Applied & Elapsed Time (Seconds) & Convergence Generation & Material Handling Cost \\
\hline \hline traditional GA & 37.877463 & 130 & 2050 \\
\hline proposed GA & 46.629072 & 100 & 1970 \\
\hline
\end{tabular}

$f_{i j}=\left[\begin{array}{llllllllll}0 & 3 & 2 & 5 & 2 & 0 & 1 & 0 & 1 & 1 \\ 3 & 0 & 3 & 2 & 3 & 0 & 1 & 3 & 2 & 1 \\ 2 & 3 & 0 & 4 & 2 & 7 & 2 & 6 & 2 & 5 \\ 5 & 2 & 4 & 0 & 1 & 5 & 3 & 1 & 3 & 1 \\ 2 & 3 & 2 & 1 & 0 & 3 & 4 & 2 & 2 & 1 \\ 0 & 0 & 7 & 5 & 3 & 0 & 5 & 0 & 3 & 5 \\ 1 & 1 & 2 & 3 & 4 & 5 & 0 & 1 & 5 & 2 \\ 0 & 3 & 6 & 1 & 2 & 0 & 1 & 0 & 3 & 1 \\ 1 & 2 & 2 & 3 & 2 & 3 & 5 & 3 & 0 & 2 \\ 1 & 1 & 5 & 1 & 1 & 5 & 2 & 1 & 2 & 0\end{array}\right]_{m_{i} \rightarrow m_{j}}$

$c_{i j}=\left[\begin{array}{llllllllll}0 & 1 & 2 & 1 & 2 & 1 & 3 & 4 & 5 & 7 \\ 1 & 0 & 2 & 2 & 4 & 1 & 8 & 2 & 2 & 6 \\ 2 & 2 & 0 & 1 & 2 & 3 & 2 & 6 & 5 & 7 \\ 1 & 2 & 1 & 0 & 1 & 1 & 3 & 1 & 5 & 1 \\ 2 & 4 & 2 & 1 & 0 & 3 & 1 & 2 & 1 & 5 \\ 1 & 1 & 3 & 1 & 3 & 0 & 5 & 1 & 3 & 1 \\ 3 & 8 & 2 & 3 & 1 & 5 & 0 & 1 & 1 & 1 \\ 4 & 2 & 6 & 1 & 2 & 1 & 1 & 0 & 3 & 1 \\ 5 & 2 & 5 & 5 & 1 & 3 & 1 & 3 & 0 & 1 \\ 7 & 6 & 7 & 1 & 5 & 1 & 1 & 1 & 1 & 0\end{array}\right]_{m_{i} \rightarrow m_{j}}$

$$
h_{i j}=\left[\begin{array}{cccccccccc}
0 & 1.2 & 2 & 1 & 0.5 & 1.2 & 2 & 1.6 & 1.8 & 1.6 \\
1.2 & 0 & 2 & 1.5 & 1.2 & 1.3 & 0.5 & 1 & 2 & 1 \\
2 & 2 & 0 & 2 & 1.6 & 1.8 & 1.2 & 0.9 & 1.3 & 1 \\
1 & 1.5 & 2 & 0 & 1.4 & 0.6 & 1.3 & 1.2 & 1.6 & 1.2 \\
0.5 & 1.2 & 1.6 & 1.4 & 0 & 1.2 & 1.5 & 1.3 & 0.8 & 0.8 \\
1.2 & 1.3 & 1.8 & 0.6 & 1.2 & 0 & 1.2 & 2 & 1 & 1.9 \\
2 & 0.5 & 1.2 & 1.3 & 1.5 & 1.2 & 0 & 2 & 1.6 & 0.6 \\
1.6 & 1 & 0.9 & 1.2 & 1.3 & 2 & 2 & 0 & 0.6 & 1.5 \\
1.8 & 2 & 1.3 & 1.6 & 0.8 & 1 & 1.6 & 0.6 & 0 & 2 \\
1.6 & 1 & 1 & 1.2 & 0.8 & 1.9 & 0.6 & 1.5 & 2 & 0
\end{array}\right]_{m_{i} \rightarrow m_{j}}
$$

\section{COMPUTATIONAL RESULT AND DISCUSSION}

Randomly generate an initial population of chromosome with POPSIZE. The genetic parameters used in this case are gen $=200, P_{c 1}=0.01, P_{c 2}=0.2, P_{m l}=0.06, P_{m l}=0.1$ per population, $\alpha_{1}=0.6, \alpha_{2}=0.4, \beta=0.5, h_{i 0}=[1,1.2,1.5,1.9,2,1,4$,$1.2,1.2,1,0.9], \Delta w=1.5, \Delta w_{0}=2, \mathrm{r}=20$, this paper present 4 simulation with different population of 50,100,200 and 300 . The program of GA was written in the MATLAB 7.9.0(R2009b).

The comparison of simulation results with elapsed time, convergence generation and material handling cost are indicated in Table 2 to Table 5. The comparisons of the object with the progress of proposed GA and traditional GA with different populations shows in Fig. (4), to Fig. (7). It is clear that the proposed GA use a little more time but has a quicker convergence speed and lower material handling cost. 


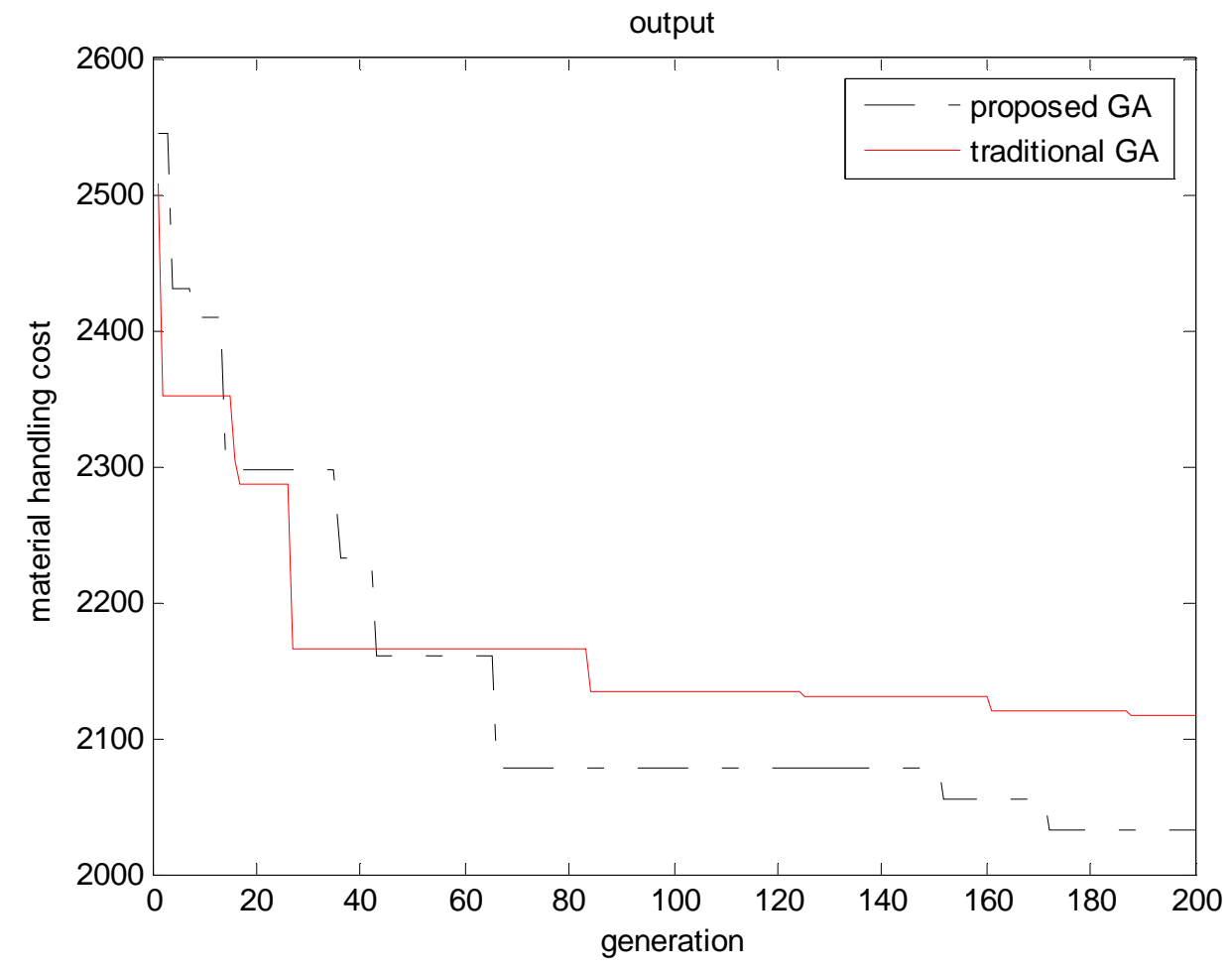

Fig. (4). Output with POPSIZE=50.

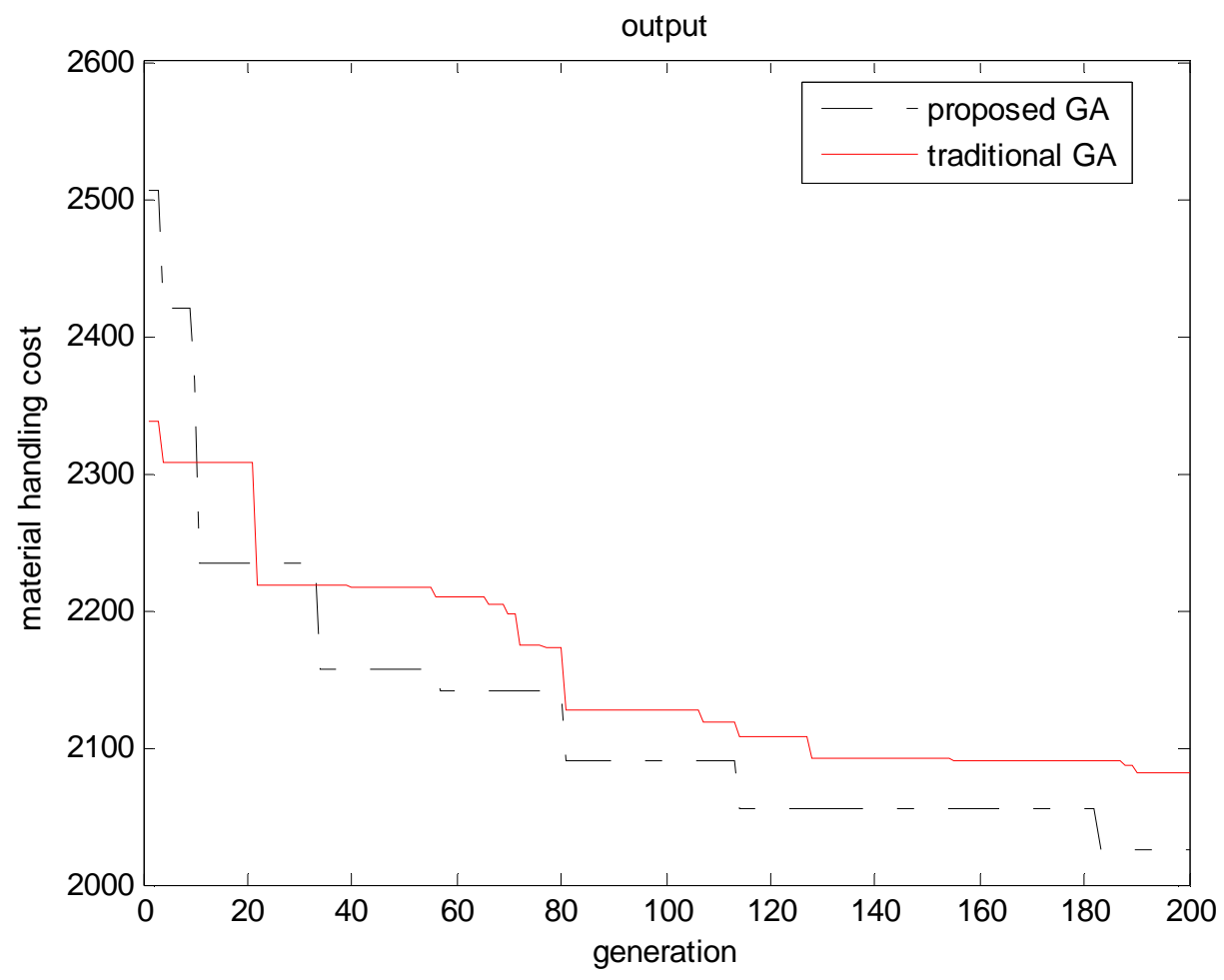

Fig. (5). Output with POPSIZE=100. 


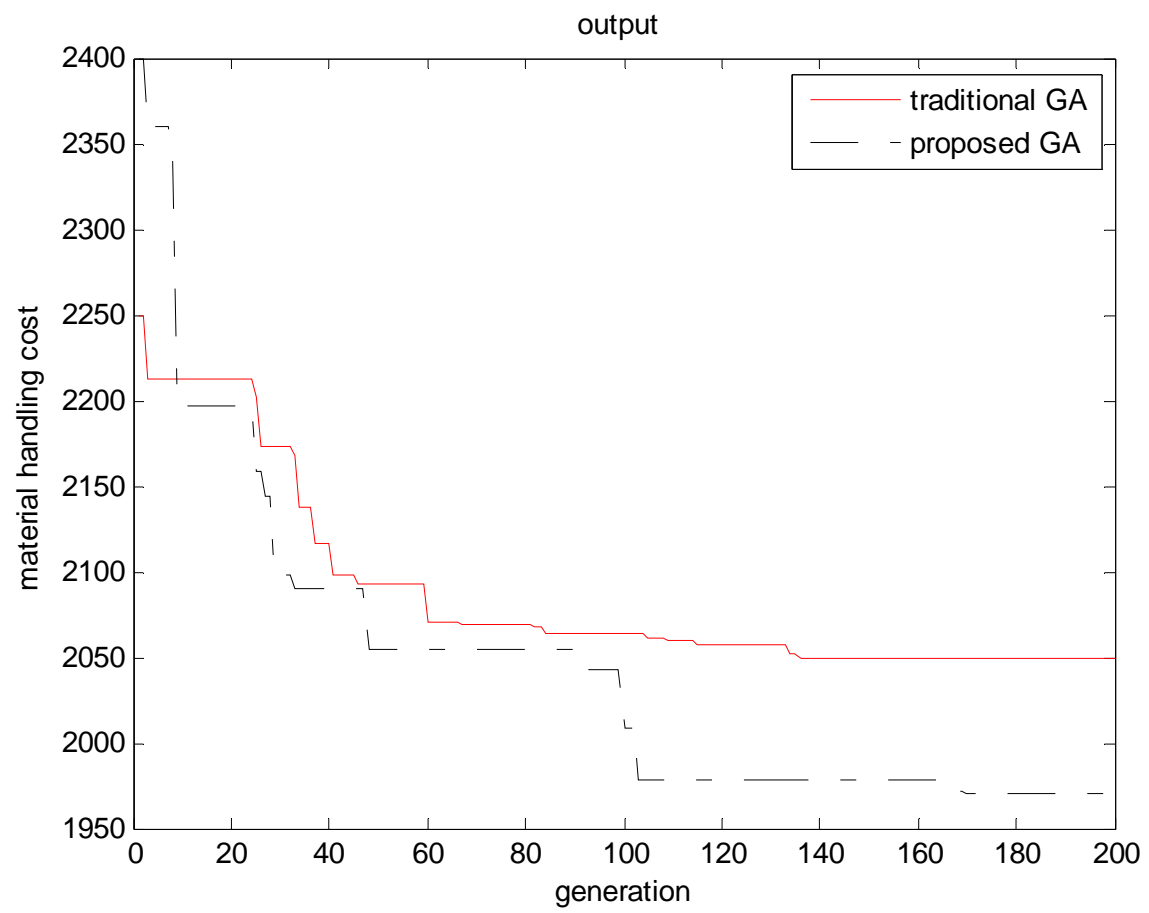

Fig. (6). Output with POPSIZE=200.

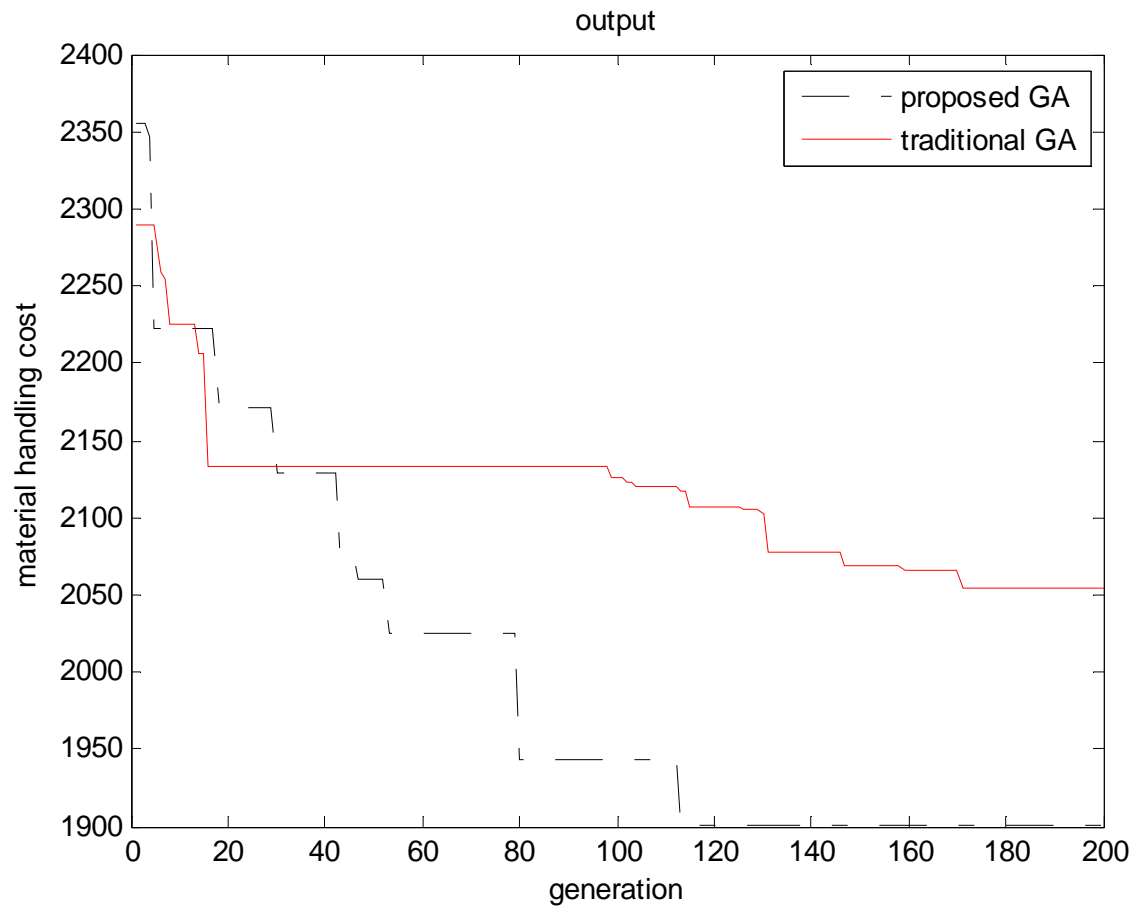

Fig. (7). Output with POPSIZE=200.

\section{CONCLUSION}

This paper proposed an approach using improved adaptive genetic algorithm to solve facility layout problems in the circumstance of multi-species and variable-batch. The proposed GA is an approach to minimize the total material handling cost. This paper combined adapted GA with optimal conservation method and greedy strategy. The effectiveness of the proposed GA has been examined by an example. The results are also compared with the work of traditional GA and it indicates that the GA proposed in the paper is more efficient for the facility layout problem. With different population size, we have simulated for four times and analyze three factors which are, convergence generation and the ma- 
terial handling cost. Although all the elapsed time with different population size of the proposed GA are longer than the traditional GA's, but the convergence generation and the material handling cost of the proposed GA are all better than the traditional one. Because that the proposed GA has to calculate the fitness and keep the excellence of the good gene, it is able to explain its longer elapsed time when compared with traditional GA. In the future, we will do more research about the facility layout problem with two or more objectives and the research should pay more attention to flexible layout with more objectives taken into consideration.

\section{CONFLICT OF INTEREST}

The authors confirm that this article content has no conflicts of interest.

\section{ACKNOWLEDGMENTS}

The authors wish to thank the scholars whose related research achievements inspired us. This work was supported in part by a grant from the Humanities and Social Sciences Base of Zhejiang (No.GK090205001-25), the open foundations of the Embedded System and Service Computing of Education Ministry Key Laboratory in Tongji University, the Defense Industrial Technology Development Program (A3920110001), the National Science Foundation of China
(No: 60175016) and the Hangzhou Dianzi University (NO: KYS 091507053) are gratefully acknowledged.

\section{REFERENCES}

[1] J. D. Balakrishnan and C.H. Cheng, "A hybrid genetic algorithm for the dynamic plant layout problem", International Journal of production Economic, vol. 86, pp. 107-120, 2003.

[2] Z. Er-min and Chen Ke-lou, "Global layout design for iron and steel plants based on SLP", Industrial Engineering Journal, vol. 14, no. 3, pp. 106-109, 2011.

[3] R, Muther, "Systematic Layout Planning", 2nd ed. Boston, Massachusetts: CahnersBooks, 1973

[4] S. Sahni and T.E Gonzalez, "Complete approximation problem", Journal of Association for Computer Machinary, vol. 23, no. 3, pp. 555-565, 1976.

[5] Q.L. Yu, H Bin and S. J. Jun, "Workshop layout optimization based on genetic algorithm", Advanced Materials Research, pp. 169-173, 2012.

[6] S. Ramazan, "A simulated annealing algorithm for solving the biobjective facility layout problem", Expert Systems with Applications, vol. 38, no. 10, pp. 4460-4465, 2011.

[7] K.Y. Wong and P. C. See, "A hybrid ant colony optimization algorithm for solving facility layout problems formulated as quadratic assignment problems", Engineering Computations: International Journal for Computer-Aided Engineering and Software, vol. 27, no. 1 , pp. 117-128, 2010.

[8] S.U.O Xiaohong and L.I.U. Zhanqiang, "Modeling and solution algorithm for facility layout of manufacturing systems", Computer Integrated Manufacturing Systems, vol. 13, no. 10, pp. 1941-1951, 2007.

(C) Li et al.; Licensee Bentham Open.

This is an open access article licensed under the terms of the Creative Commons Attribution Non-Commercial License (http://creativecommons.org/licenses/by-nc/3.0/) which permits unrestricted, non-commercial use, distribution and reproduction in any medium, provided the work is properly cited. 